\title{
Physical aspects of the lithium ion interaction with the imperfect silicene located on a silver substrate
}

\author{
A. E. Galashev ${ }^{1,2, \dagger}$, K. A. Ivanichkina ${ }^{1}$, O. R. Rakhmanova ${ }^{1,2}$, Yu. P. Zaikov ${ }^{1,2}$ \\ †alexander-galashev@yandex.ru
}

\begin{abstract}
${ }^{1}$ Institute of High-Temperature Electrochemistry, Ural Branch of RAS, 20 Akademicheskaya str., Yekaterinburg, 620990, Russia ${ }^{2}$ Ural Federal University n. a. the first President of Russia B. N. Yeltsin, 19 Mir str., Yekaterinburg, 620002, Russia
\end{abstract}

\begin{abstract}
Epitaxy of $\mathrm{Si}$ on a silver substrate is the main method to obtain silicene. The latter does not separate from the substrate. In the present paper, the possibility of using silicene on a silver substrate as an anode for lithium-ion batteries is studied by the method of molecular dynamics. Structural and mechanical effects arising from the motion of a $\mathrm{Li}^{+}$ion through a planar channel formed by a perfect and defective two-layer silicene are studied. Generally, the defect stability and silicene sheet integrity are independent of the $\mathrm{Ag}(001)$ or $\mathrm{Ag}(111)$ substrate type. The transverse vibrations of $\mathrm{Si}$ atoms in the channel have a significant effect on the motion of lithium ions. This effect is taken into account by using the interference factor that describes the slowing down of the motion of the $\mathrm{Li}^{+}$ion in the channel. The dependence of this coefficient on the size of vacancy defects in silicene is determined. The presence of the substrate makes this dependence less relevant. The stress distribution in the defective silicene while driving a lithium ion along the planar silicene channel is calculated. The strongest stresses in the silicene are created by forces directed perpendicular to the strength of the external electric field. These forces dominate in the silicene channel placed on the substrates of both types.
\end{abstract}

Keywords: silicene, structure, molecular dynamic, substrate.

\section{Introduction}

Silicene is a monolayer of silicon atoms forming a hexagonal honeycomblattice. In fact, the lattice consists of two sublattices with a very weak division. Silicene is a topological insulator. It is characterized by a fully insulating gap in the central part of its band structure and the presence of gapless helical edges. The phase transition from a topological insulator to a zone dielectric is performed by applying the external electric field. Although silicene is supposed to be a semiconductor without a bandgap due to the $s p^{2}$-hybridization of Si atoms, a slight overlap between $3 p$-orbitals results in the formation of a reactive surface that is energetically more stable at the adsorption of other chemicals.

Lithium-ion batteries are widely used as power sources due to the high energy density, relatively high voltages, and a low weight to volume ratio. Simulation together with experimental test makes it possible to develop a wide variety of battery designs covering a wide range of operating conditions at low cost.

The energy density depends heavily on the specific capacity of the electrode. Anodes based on silicon draw special attention due to their high theoretical capacity $(4200 \mathrm{mAh} / \mathrm{g})$ as compared with $372 \mathrm{mAh} / \mathrm{g}$ for graphite [1]. The main disadvantage of silicon used in anode structures is its swelling during lithiation [2]. It results in the anode destruction and consequent reduction of the charge rate. A possible way to avoid that is to use two-dimensional multi-layer materials such as graphene and silicene. Studies on the electronic structure of totally lithiated silicene have shown that there is a transformation of the band structure of a zero-band gap semiconductor into the structure of a semiconductor with the band gap of $0.37 \mathrm{eV}$. This feature distinguishes silicene from graphene which exhibits metal properties during lithiation [2]. After total delithiation silicene restores its original properties in contrast to the crystalline Si that experiences sufficient structural changes. The observed low energy barrier for diffusion of Li on silicene regardless of the concentration of adsorbed Li atoms means that silicene is a suitable material for anodes of lithium-ion batteries [3].

Defects are almost inevitable during the fabrication process and have a pronounced effect on different properties of silicene. The atomistic mechanisms in the self-healing of vacancy defects in free-standing silicene are considered in theoretical work [4]. The structures, formation energy, and migration behavior of typical mono- and bivacancies are studied in theoretical work [5]. Experimental studies provide strong evidence that a large number of defect clusters with more than three missing silicon atoms are observed in the scanning tunneling microscope images [6]. The mechanism of vacancy transformation is studied using the density functional theory (DFT) [7]. A monovacancy obtained by removing one Si atom transforms to 5-fold and 9-fold rings. Defects (vacancies) can influence significantly the ion ability to move along the silicene channel. Also, there is an energy barrier for the lithium ion to enter the silicene channel. $A b$ initio calculations show that the energy barrier for lithium ion to penetrate the free-standing silicene is $0.6 \mathrm{eV}$ [8]. 
According to the MD calculations [9], lithium ions with the initial energy of $0.5-2.0 \mathrm{eV}$ can easily enter the silicene channel with graphene support with the gap size not less than $0.75 \mathrm{~nm}$.

The objective of this work is to study the physical properties of bilayer porous silicene on $\mathrm{Ag}(001)$ and $\mathrm{Ag}(111)$ substrates in the process of silicene interaction with a lithium ion in a constant electric field.

\section{Computer model}

The calculations are made using the method of classical molecular dynamics simulation. Interatomic interactions in silicene are described by the many-body Tersoff potential [10]. The interaction between $\mathrm{Si}$ atoms belonging to different silicene sheets is described by the Morse potential [11]. The Morse potential is also used to represent the interactions between $\mathrm{Li}^{+}$ion and $\mathrm{Si}$ or Ag atoms [12-13].

In this work, a $4 \times 4$ surface reconstruction is studied. A unit cell of such silicene structure contains $18 \mathrm{Si}$ atoms. Six $\mathrm{Si}$ atoms are normally displaced by $0.065 \mathrm{~nm}$ to the surface; the other Si atoms are located on the same (initial) plane. The initial structure of a silicene sheet is similar to the silicene surface experimentally observed on the $\mathrm{Ag}(111)$ substrate [14]. In the case of longitudinal movement of $\mathrm{Li}^{+}$ion, the values of $0.75,0.80$ and $0.85 \mathrm{~nm}$ of gap $h_{g}$ between silicene sheets are used.

Perfect silicene sheets have sizes of $4.8 \times 4.1 \mathrm{~nm}$ (taking into account the size of $\mathrm{Si}$ atoms) and contain 300 atoms. Nine mono- and polyvacancies (bi-, tri- and hexavacancies) are arranged roughly uniformly over the silicene sheet area. The number of Si atoms in every sheet is $291,282,273$, and 246 for mono-, bi-, tri- and hexavacancies, respectively. The following designations: II - monovacancies, III - bivacancies, IV - trivacancies, V - hexavacancies are used for systems that are perfect (I) or have defects in silicene. The silicene sheets are arranged according to the Bernal packing $(A B A B \ldots)$ in exactly the same way as in the bulk graphite. Bilayer silicene is placed on unreconstructed (001) and (111) surfaces of the FCC silver crystal. Ag atoms are 3.84 times heavier than Si ones. Consequently, Ag atoms should acquire a lower speed per the same number of times when colliding with $\mathrm{Si}$ atoms. Therefore, with reasonable accuracy in calculations, the approach of interacting but not moving $\mathrm{Ag}$ atoms is used. A series of additional experiments were carried out to check the reliability of the obtained results. Three rounds of calculations for every system were simulated. The calculations showed a reliable reproducibility of the results, the error in determining the passage time of the ion through the silicene channel is $20 \%$.

The bond length determined between the first-layer $\mathrm{Si}$ and $\mathrm{Ag}$ atoms is $0.27 \mathrm{~nm}$. It is close to the average values for $\mathrm{Si}-\mathrm{Si}(0.235 \mathrm{~nm})$ and $\mathrm{Ag}-\mathrm{Ag}(0.289 \mathrm{~nm})$ bonds in bulk crystals [15]. This distance was determined by the electron diffraction. The measurements were performed for the Ag film deposited on the $\mathrm{Si}(111)$ surface. At $d_{\mathrm{Si} A \mathrm{~g}}=0.27 \mathrm{~nm}$, the silicon film on the Ag substrate undergoes substantially smaller perturbations induced by the action of the metal substrate than at $d_{\mathrm{Si}-\mathrm{Ag}}=0.247 \mathrm{~nm}$ obtained in the DFT calculation [16]. Therefore, the initial value of $d_{\mathrm{Si}-\mathrm{Ag}}=0.27 \mathrm{~nm}$ was chosen.
Fixing of the edges of the silicene sheets permits to maintain the morphology of the porous silicene. The simulation time step is $\Delta t=1 \times 10^{-16} \mathrm{~s}$. The duration of the calculations for every vacancy type is 1 million time steps, or 100 ps. All calculations are performed in the NVT ensemble. The ion is moved horizontally along the channel by the electric field with the strength vector directed along the $o x$ axis. The electric field strength used in this study is $10^{6} \mathrm{~V} / \mathrm{m}$. According to the additional calculations, if there is no external electric field in the system, the lithium ion experience thermal vibrations and is fixed in the most energetically favorable position in the channel. It is opposite to the center of the six-membered ring of the closest silicene sheet.

To calculate stresses arising in silicene, every sheet is divided into elementary areas. Stresses $\sigma_{u x}(l)$ caused by the forces in the $\alpha(\alpha=x, y, z)$ direction are calculated for each $l$-th area with $u$ orientation. In these calculations the products of projections of atomic velocities and projections of forces $f_{i j}^{\alpha}$ acting on the $l$-th area from the direction of other atoms are used if the corresponding conditions are satisfied [17]

$$
\sigma_{u \alpha}(l)=\left\langle\sum_{i}^{k} \frac{1}{\Omega}\left(m v_{u}^{i} v_{\alpha}^{i}\right)\right\rangle+\frac{1}{S_{l}}\left\langle\sum_{i}^{k} \sum_{j \neq i}^{\left(u_{i}<u, u_{j} \geq u\right)}\left(f_{i j}^{\alpha}\right)\right\rangle .
$$

Here $k$ is the number of atoms in the $l$-th area, $\Omega$ is volume per atom, $m$ is the atomic mass, $v_{\alpha}^{i}$ is the $\alpha$-projection of the $i$-th atom velocity, and $S_{l}$ is the $l$-th area. The conditions of summation over $j$ in the last sum of expression (1) are reflected in both the lower and upper indexes of the sum; the force arising during the interaction of atoms $i$ and $j$ passes through the $l$-th area; $u_{i}$ is the current coordinate of atom $i$; in the upper bound of the sum $u$ is the coordinate of the point of contact of the straight line passing through the centers of atoms $i$ and $j$ with the $l$-th area.

A modified version of the LAMMPS program was used for simulation [18]. The calculations were performed using the "URAN" cluster-type hybrid computer at the Institute of Mathematics and Mechanics of UB of RAS.

\section{Results and discussion}

Movement of $\mathrm{Li}^{+}$ion along the silicene channel located on the $\operatorname{Ag}(111)$ surface is shown in Fig. 1. In this case, the silicene sheets have no vacancies and the gap between them is $0.85 \mathrm{~nm}$. As a result of interaction between the silver substrate and both the top and bottom silicene sheet the latter assume a convex shape. The channel narrows considerably. The interaction between the ion and both silicene sheets leads to an oscillating ion trajectory. The movement of the ion is not strictly vibrational-translational. The ion has a trajectory over the entire area of the silicene sheets including reverse movement. Since the silver substrate can have an occasional effect, the lithium ion path can be long or short. An example of a short ion path is represented on the right of Fig. 1.

To analyze the time it takes the lithium ion to pass through the channel interference factor $K_{\text {int }}$ was introduced. If the total energy of system $E_{t o t}$ (characterizing the sum of longitudinal and transverse oscillations) and the energy of $\mathrm{Si}-\mathrm{Si}$ interactions $E_{\mathrm{Si}-\mathrm{Si}}$ inside the sheets (longitudinal oscillations in the silicene sheets) are known, 
then the interference factor can be calculated as follows: $K_{\text {int }}=\left(E_{\text {tot }}-E_{\mathrm{Si}-\mathrm{Si}}\right) / E_{\mathrm{Si}-\mathrm{Si}}$. It characterizes the effect of transverse oscillations in the system on the lithium ion movement. In the case of the «ion+free-standing silicene» system, the monotonic increase of factor $K_{i n t}$ with the vacancy size was obtained (Fig. 2a, blue curve). The lithium ion time of passage also increases monotonically (Fig. 2b). The bigger the vacancy size, the more slowly the ion moves along the channel. That lays emphasis on the fact that the vacancy size correlates with the ion time of passage in the system under consideration. When the silicene is located on the Ag substrate the factor $K_{\text {int }}$ consistently increases with increasing defect size. As a result of the increased interference, the irregular fluctuations of lithium ion time of passage through the channel can be observed (Fig. 2b, green and red curves). Transverse oscillations are of greater importance in such systems.

Fig. 3 shows a horizontal projection of the bottom silicene sheet with monovacancies located on the $\operatorname{Ag}(001)$ substrate. It represents how the shape of the defects in the horizontal plane is changed. The change occurs due to the movement of the ion along the channel. The contours of the initially formed defects are shown at the top of Fig. 3. It can be seen that three of the nine previously presented defects are disintegrated after 100 ps. Their transformation is shown by the circle and the ellipse. The circle represents the formation of five-fold rings while the ellipse shows the formation of rings normally containing $9 \mathrm{Si}$ atoms. An even more complex destruction is observed for the defects of a bigger size.

The average lithium ion time of passage through the channel can vary from several ps up to tens of ps depending on the vacancy size. When the ion moves along the channel, it makes an oscillating motion of large amplitude between the silicene sheets. When moving, the ion interacts with silicene most closely and can cause significant damage to the silicene sheets [19]. When the ion leaves the channel, the shape of vacancies does not change dramatically. Only the channel shape can be modified during remaining time (to a greater extent, it returns to the original shape).
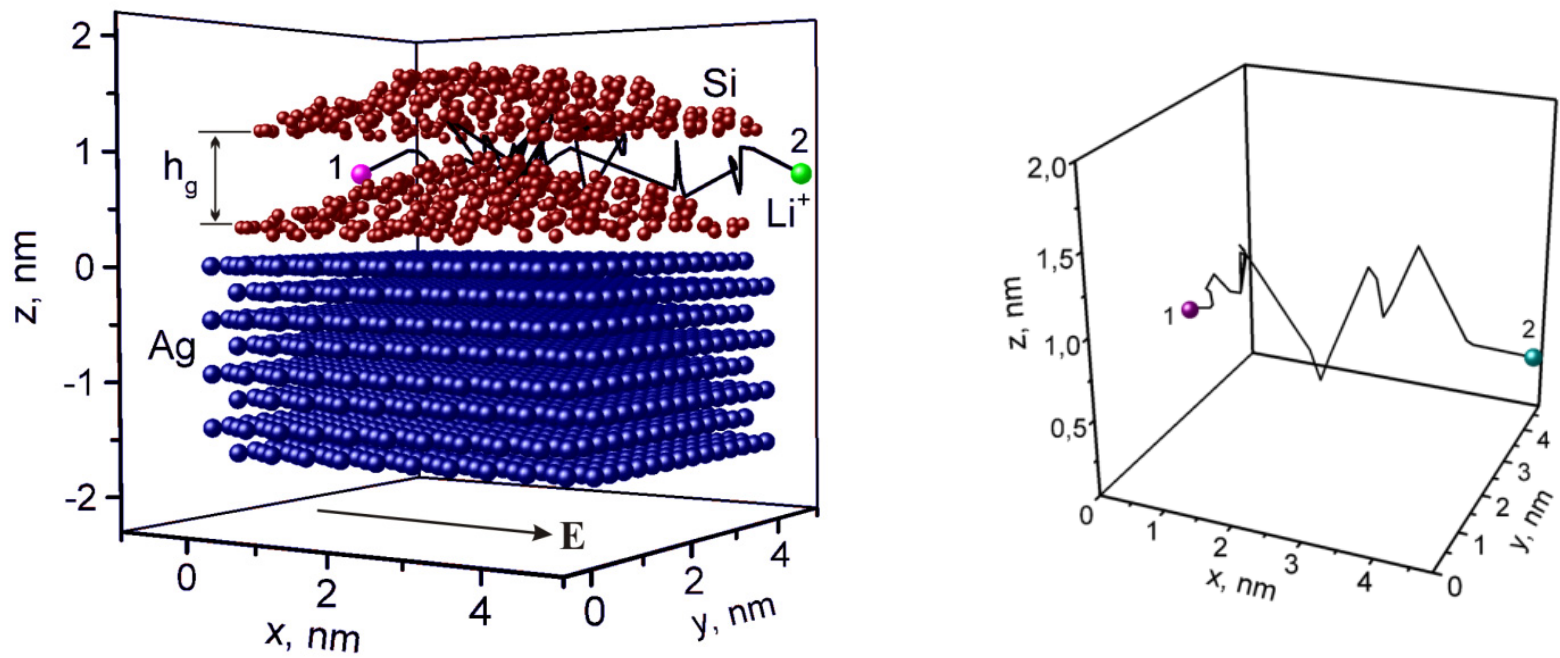

Fig. 1. The path passed by $\mathrm{Li}^{+}$ion in the silicene channel supported by $\operatorname{Ag}(111)$ substrate in the constant electric field with the intensity $E$ (on the left) and the example of a short lithium ion path (on the right): 1 and 2 are the initial and final points of the ion trajectory, respectively.
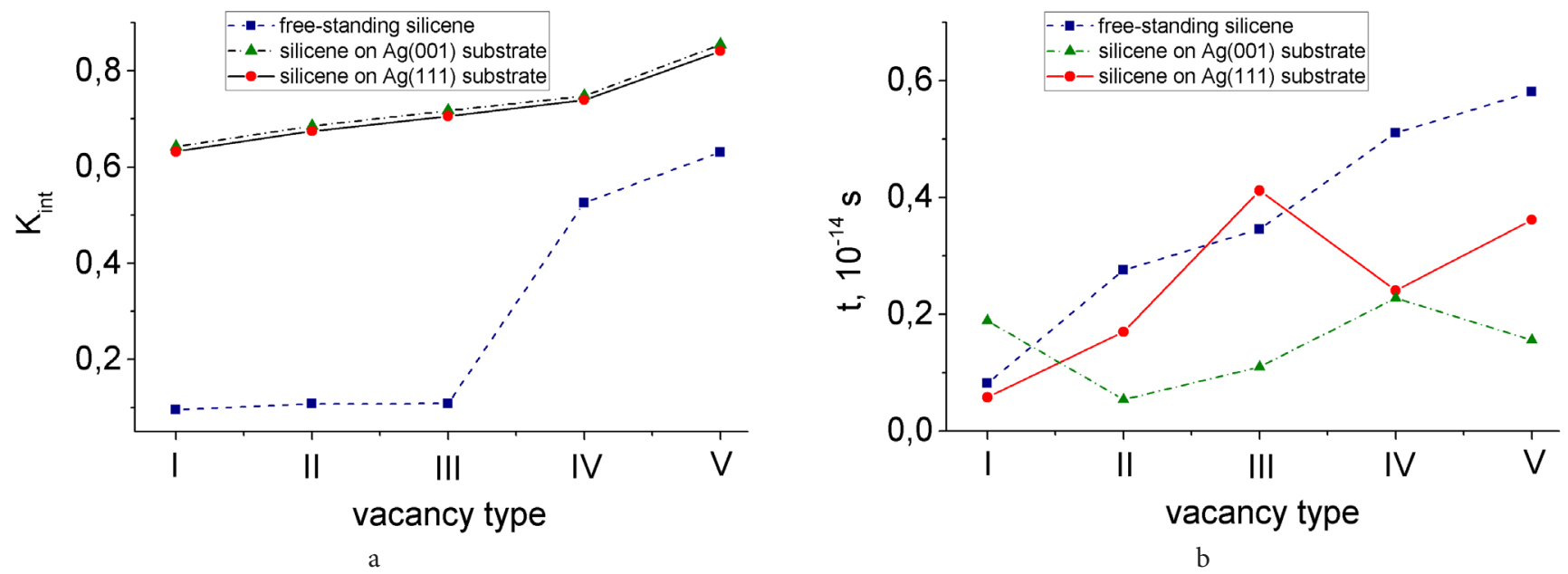

Fig. 2. (Color online) Interference factor (a) and lithium ion time of passage through channel (b) for the systems: free-standing silicene (blue); silicene on $\operatorname{Ag}(001)$ substrate (green); silicene on $\operatorname{Ag}(111)$ substrate (red) depending on the vacancy type of the silicene; I is a perfect silicene and II - V are the silicene sheets with: II mono-, III bi-, IV tri- and V hexavacancies. 
In all the cases under consideration, the local $\sigma_{z z}$ stresses induced by the forces of the vertical direction (acting perpendicular to the silicene surface) are not dominant. The more significant local $\sigma_{z y}$ stresses arise in the silicene plane. They are induced by the forces acting in the plane perpendicular to the direction of the electric field vector. Such scheme of stresses distribution is observed in the bottom silicene sheet with trivacancies located on the $\mathrm{Ag}(001)$ substrate (Fig. 4). More intense peaks of stresses in the bottom silicene sheet are observed in its middle part $(2.3-3.8 \mathrm{~nm})$. This part of silicene sheets is exposed to a greater deformation. The peaks of local stresses arise due to the movement of the ion. The middle part of the sheets corresponds to the narrowest area of the deformed channel. $\mathrm{The}^{\mathrm{Li}} \mathrm{i}^{+}$ion creates great stresses in this area while passing through.

It should be noted that the local stresses, in this case, is a microscopic characteristic. It is calculated as a ratio of the acting force applied to the elementary area. The sign of the direction of the force determines the sign of the stresses. The value of the $\sigma_{z y}$ component can be permanently negative due to the predominant location of the ion above the grooves formed by the equally directed rows of $\mathrm{Si}$ atoms.

\section{Conclusion}

The motion of lithium ion in the constant electric field along the flat channel formed by the defective silicene sheets has been studied using the molecular dynamics method. Polyvacancies in silicene account for a number of useful properties including the band gap expanding, ferromagnetism, etc. However, when using silicene as an anode material, polyvacancies are likely to have a negative effect. This is due to the difficultly a $\mathrm{Li}^{+}$ion experiences when passing through the flat channel formed by the sheets of the defective silicene. Moreover, an uncontrolled motion of the ion migrating through the channel in the electric field is under way. In addition, defects such as vacancies weaken the strength of silicene and reduce its mechanical stability. The presence of the silver substrate does not improve the properties, but on the contrary, worsen them. As a result of interaction with metal substrate, the vertical displacements of atoms in silicene are increased, the probability of $s p^{3}$-hybridization increases, and the mechanical stability reduces. The stresses induced by the forces in the silicene plane and acting perpendicularly to the direction of the strength vector of the electric field can exceed significantly the stresses induced by forces acting along the field or in the direction perpendicular to the plane of the silicene.

Taking in to account the results obtained, it can be concluded that the use of silicene on the $\operatorname{Ag}(100)$ and $\mathrm{Ag}(111)$ substrates as an anode of lithium-ion battery is ineffective and expensive. In prospect, it is planned to find a more suitable substrate for a two-layer silicene to be used as an anode material.

Acknowledgements. This study is supported by the Russian Science Foundation (project no. 16-13-00061).

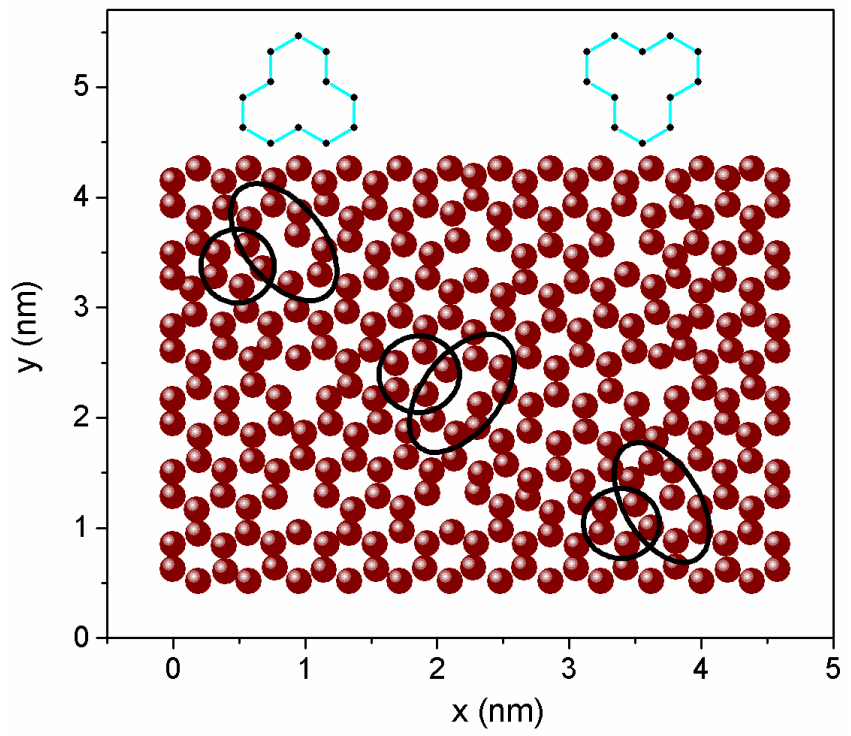

Fig. 3. The horizontal projection of the bottom silicene sheet located on the $\operatorname{Ag}(001)$ substrate and corresponding to the time moment of 100 ps. The contours of the initially created monovacancies are shown above (cyan); the transformed monodefects are marked by circles and ellipses.

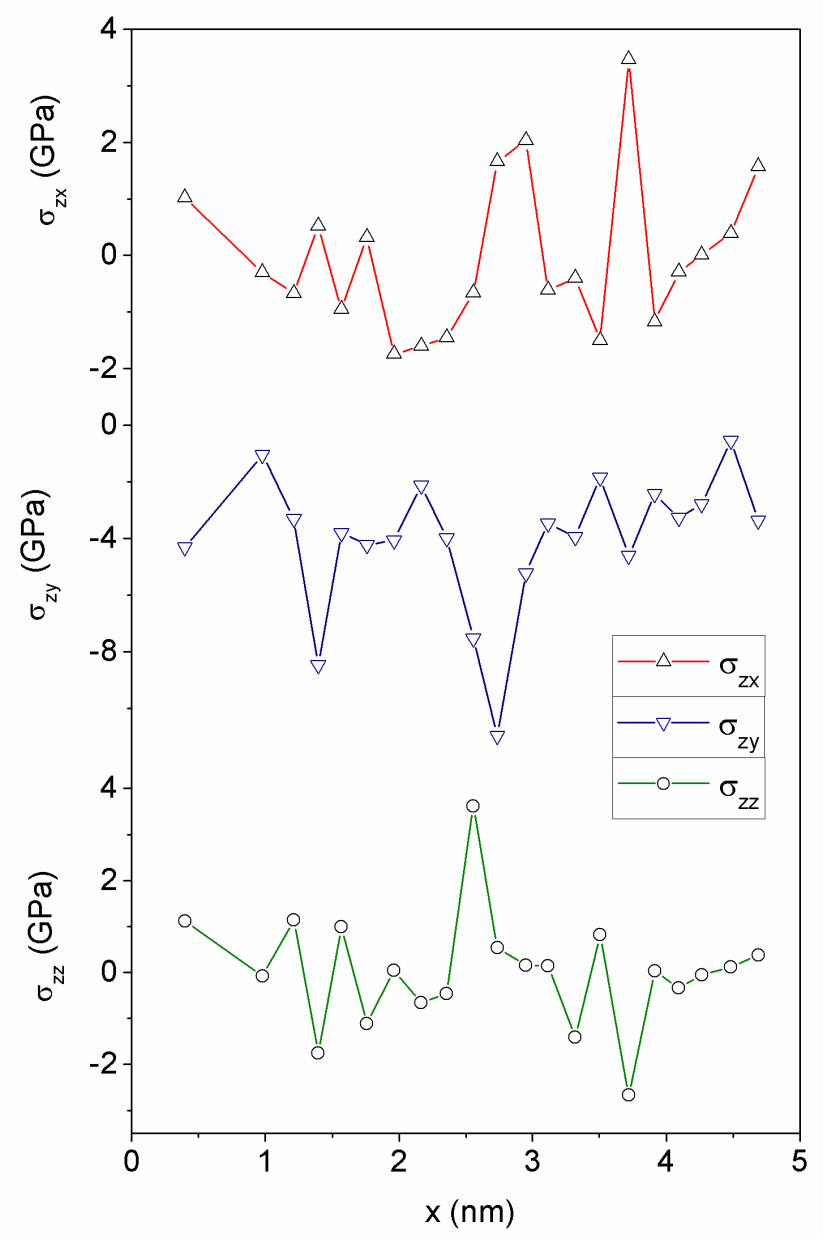

Fig. 4. (Color online) The stresses distribution in the bottom silicene sheet with trivacancies obtained while $\mathrm{Li}^{+}$ion moves under the constant electric field along the flat channel located on the $\operatorname{Ag}(001)$ substrate: $\sigma_{z x}$ (red), $\sigma_{z y}$ (blue), $\sigma_{z z}$ (green). The field strength vector is directed along the $o x$ axis. 


\section{References}

1. G. A. Tritsaris, E. Kaxiras, S. Meng, E. Wang. NanoLett. 13(5), 2258 (2013). DOI: 10.1021/nl400830u

2. G.A. Tritsaris, K. Zhao, O.U. Okeke, E. Kaxiras. J. Phys. Chem. C. 116(42), 22212 (2012). DOI: $10.1021 /$ jp307221q

3. J. Huang, H.-J. Chen, M.-S. Wu, G. Liu, C.-Y. Ouyang, B. Xu. Chin. Phys. Lett. 30(1), 017103(1-5) (2013). DOI: $10.1088 / 0256-307 X / 30 / 1 / 017103$

4. V.O. Ozcelik, H.H. Gured, S. Ciraci. Phys. Rev. B. 88(4), 045440(1-6) (2013). DOI: 10.1103/PhysRevB.88.045440

5. J.F. Guo, J.F. Zhang, H.S. Liu, Q.F. Zhang, J.J. Zhao. Nanoscale 5(20), 9785 (2013). DOI: $10.1039 / \mathrm{C} 3 \mathrm{NR} 02826 \mathrm{G}$

6. H. Jamgotchain, Y. Colignon, B. Ealet, B. Parditka, J.-Y. Hoarau, C. Girardeaux, B. Aufray, J.-P. Bibérian. J. Phys.: Conf. Ser. 491(1), 012001(1-7) (2014). DOI: 10.1088/1742-6596/491/1/012001

7. G. R. Berdiyorov, F.M. Peeters. RSC Adv. 4(3), 1133 (2014). DOI: 10.1039/C3RA43487G

8. G. A. Tritsaris, E. Kaxiras, S. Meng, E. Wang. NanoLett. 13(5), 2258 (2013). DOI: 10.1021/nl400830u

9. O. R. Rakhmanova, A. Y. Galashev. Rus. J. Phys. Chem. A.
91(5), 921 (2017). DOI: 10.1134/S003602441705020X

10. J. Tersoff. Phys. Rev. B. 49(23), 16349 (1994). DOI: 10.1103/PhysRevB.49.16349

11. R. Yu, P. Zhai, G. Li, L. Liu. J. Electron. Mater. 41(6), 1465 (2012). DOI: 10.1007/s11664-012-1916-X

12. S. K. Das, D. Roy, S. J. Sengupta. Phys. F: Metal. Phys. 7(1), 5 (1977). DOI: 10.1088/0305-4608/7/1/011

13. K.-N. Chiang, C.-Y. Chou, C.-J. Wu, C.-J. Huang, M.-C. Yew. Comp. Modell. Eng. Sci. 37(1), 85 (2008). DOI: $10.3970 / \mathrm{cmes} .2008 .037 .085$

14. T. Shirai, T. Shirasawa, T. Hirahara, N. Fukui, T. Takahashi, S. Hasegawa. Phys Rev B. 89(24), 241403(1-6) (2014). DOI: $10.1103 /$ PhysRevB.89.241403

15. S. Kono, H. Sakurai, T. Sagawa, Surf. Sci. Lett. 130(1), L299 (1983). DOI: 10.1016/0167-2584(83)90596-0

16. Y.-P. Wang, H.-P. Cheng, Phys. Rev. B. 87(24), 245430 (2013). DOI: 10.1103/PhysRevB.87.245430

17. A.E. Galashev. Phys. Met. Metallogr. 117(3), 246 (2016). DOI: 10.1134/S0031918X16030054

18. S. J. Plimpton. Comp. Phys. 117(1), 1 (1995). DOI: $10.1006 /$ jcph.1995.103

19. A.E. Galashev, Yu.P. Zaikov, R.G. Vladykin. Rus. J. Electrochem. 52(10), $966 \quad$ (2016). DOI: $10.1134 /$ S1023193516100049 